\title{
Spectral Deconvolution and Quantification in EDS Using Low Energy X-ray Lines From Steel Spectra
}

\author{
Ralf Terborg $^{1}$, Tobias Salge ${ }^{2}$, Philippe T. Pinard ${ }^{3}$ and Silvia Richter ${ }^{3}$ \\ ${ }^{1 .}$ Bruker Nano GmbH, Am Studio 2D, 12489 Berlin, Germany \\ 2. Core Research Laboratories, Natural History Museum, London, UK \\ 3. Central Facility for Electron Microscopy, RWTH Aachen University, Aachen, Germany.
}

One of the most important materials used in industry is steel. Its fine microstructure consisting of different phases and inclusions, has led to the development of new steel alloys that push the analytical requirements for spatial resolutions down to the $100 \mathrm{~nm}$ scale. In X-ray microanalysis either using energy or wavelength dispersive spectrometry (EDS, WDS), low accelerating voltages, e.g. $\leq 6 \mathrm{kV}$, can fulfill this requirement. At low accelerating voltages only the $\mathrm{L}$ lines for most steel alloying elements (e.g. $\mathrm{Cr}, \mathrm{Mn}, \mathrm{Fe}, \mathrm{Co}, \mathrm{Ni}$ ) can be determined as their $\mathrm{K}$ lines can no longer be excited. Qualitative and quantitative analysis of low energy L lines is very challenging due to the presence of absorption edges within the bremsstrahlung background, the energy dependence of the efficiency and the uncertainties of absorption effects [1]. Peak overlaps, furthermore are considerable for EDS and can even be significant for WDS.

Due to these effects this type of analysis poses difficulties for EDS and WDS. To evaluate and compare these challenges for both techniques, a systematic study was performed on steels at acceleration voltages of $15 \mathrm{kV}$ and $5-6 \mathrm{kV}[1,2]$. Spectra and net intensities of 15 steel samples covering a wide range of concentrations of the major elements were simultaneously acquired on a JEOL JXA8530F electron microprobe equipped with 5 WD spectrometers and a Bruker XFlash ED spectrometer.

The results of the $15 \mathrm{kV}$ spectra show that for elements with a concentration of $<1 \mathrm{wt} . \%$ the WDS results are more accurate when significant peak overlaps occur in ED spectra ( $\mathrm{Mn}, \mathrm{Co}, \mathrm{Cu})$. For elements without peak overlaps, EDS results were comparable ( $\mathrm{Si}, \mathrm{Mo}$ ) to WDS [2]. At an acceleration voltage of $6 \mathrm{kV}$, the deconvolution results of ED spectra show agreement with the experimental spectra, fig. 1. Since the quantification of $\mathrm{L}$ lines is more sensitive to the quantification procedure and fundamental parameters (e.g. mass absorption coefficient) used, the comparison focuses on k-ratios instead of the quantified mass fractions. Fig. 2 shows a good agreement between EDS and WDS kratios, substantiating the peak deconvolution procedure shown in fig. 1.

The aim of this study is to evaluate the options of line net count determination by peak deconvolution or peak fitting and the dependence on the background removal.

References:

[1] Llovet et al, IOP Conf. Series: Materials Science and Engineering 32, 2012, 012014

[2] PT Pinard et al, Micosc. Microanal. 21 (Suppl 3), 2015, 1879 


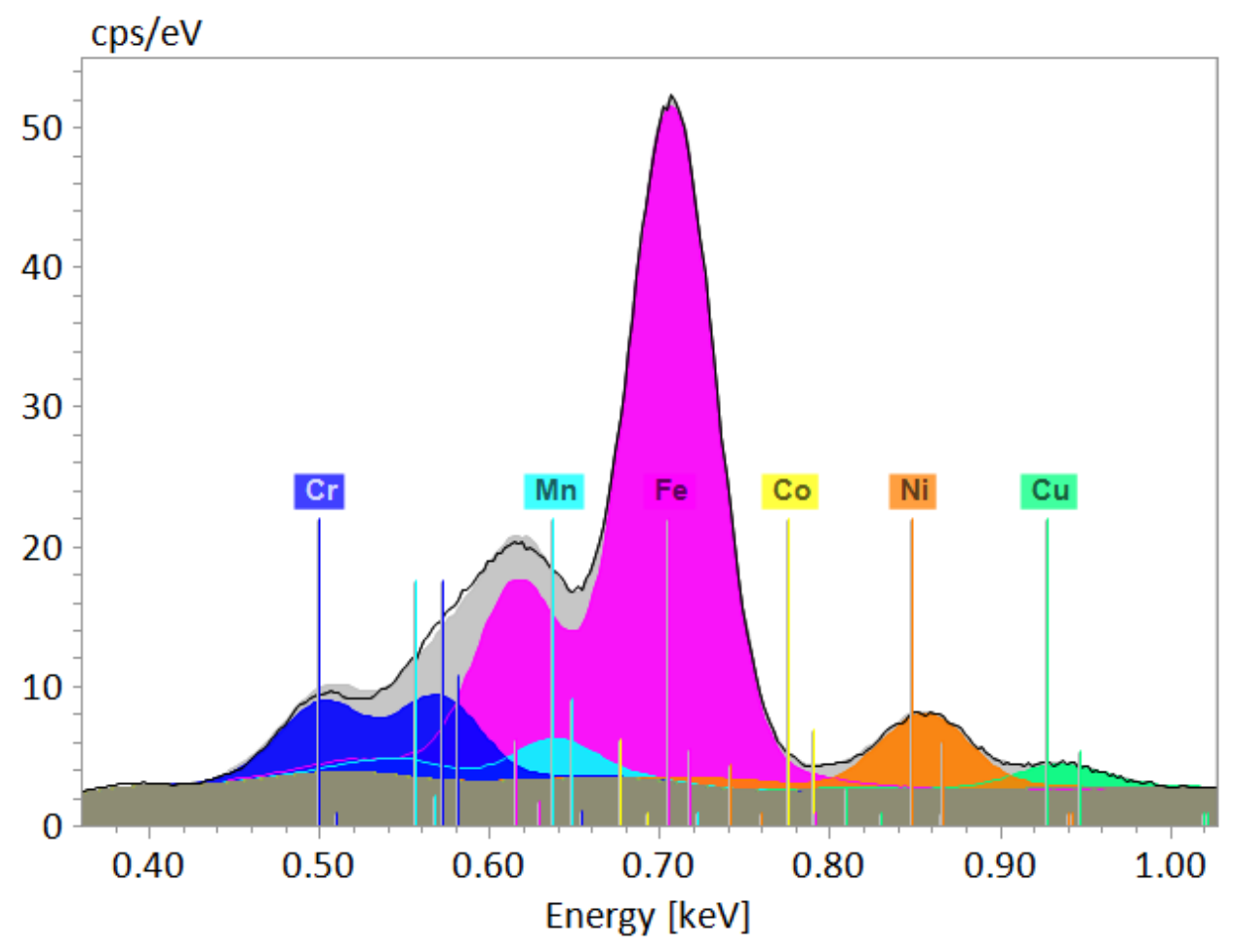

Figure 1. Deconvolution result of the $\mathrm{L}$ lines of $\mathrm{Cr}, \mathrm{Mn}, \mathrm{Fe}, \mathrm{Co}, \mathrm{Ni}, \mathrm{Cu}$ in an EDS spectrum from a steel sample, acceleration voltage $6 \mathrm{kV}$. The black line shows the experimental spectrum, the dark grey area shows the bremsstrahlung background, the light grey area shows the superposition of the element line profiles.

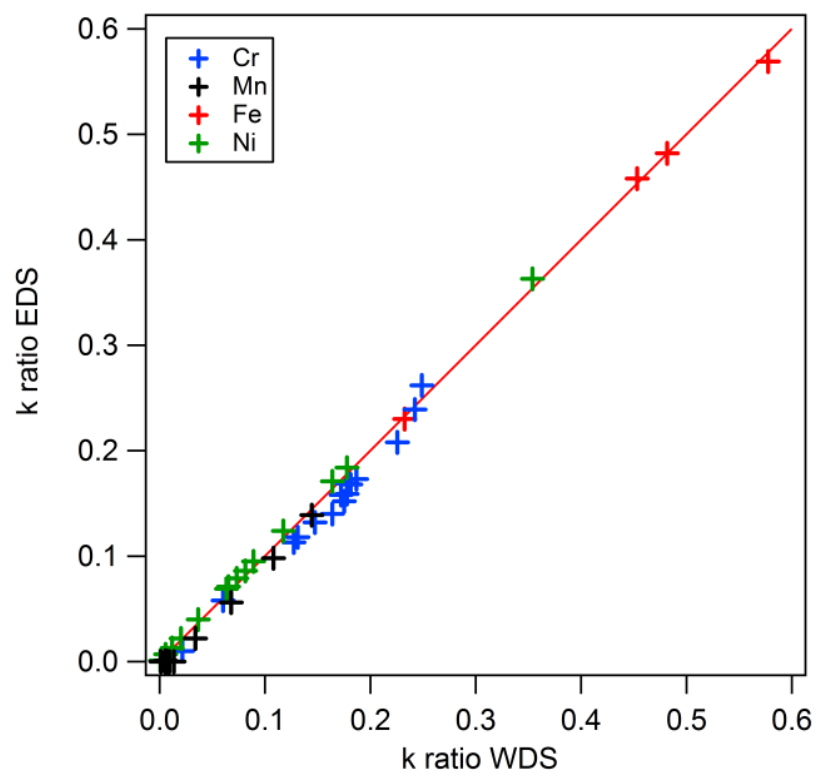

Figure 2. Graph of k ratios from EDS measurements versus WDS k ratios of 15 steel samples in order to compare the performance of the spectrometers. 